\title{
Electron Accelerator Recycles Energy for a Brighter Beam
}

\author{
A test electron accelerator reuses the energy of the particles in order to \\ achieve a brighter beam without drawing more power from the grid.
}

By Jessica Thomas

inear particle accelerators (linacs) are the machines of choice for dense beams with precise energies. But compared with their circular counterparts, they have a waste problem: After one trip, the beam-and the enormous amount of energy needed to accelerate it-are lost. Researchers at Cornell University and Brookhaven National Laboratory (BNL), both in New York, have now tested a linac for electrons that recovers $99.8 \%$ of an existing beam's energy to accelerate new particles. Although other recovery linacs exist, the machine's innovative design would enable brighter beams for less cost.

Particles in an accelerator get their energy by passing through long metal tanks, or "cavities," that house intense oscillating electromagnetic fields. A power source has to continually supply the fields. But an already accelerated beam can also replenish a field, if it passes through the cavity at the right time

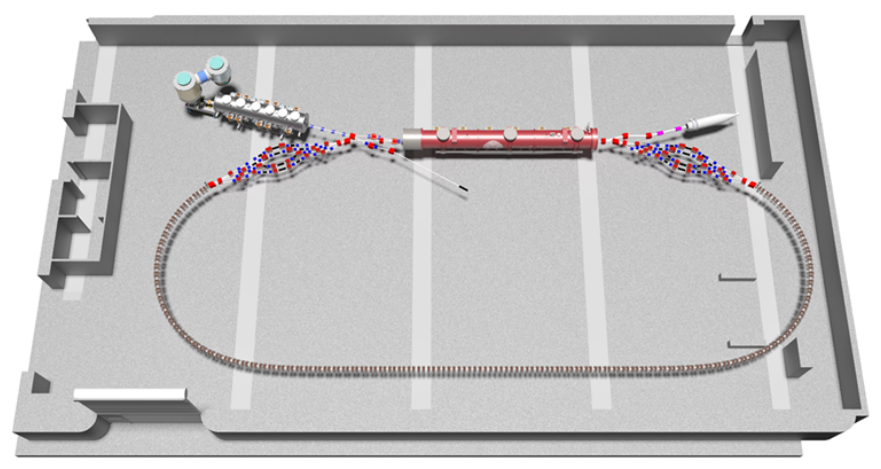

Credit: CLASSE in the field's oscillation cycle.

The new machine, CBETA, incorporates energy recovery and other energy-saving technologies. The cavities have superconducting walls, allowing for long-time field storage. The beam is looped through the same linac multiple times, first with four passes to accelerate and then four passes to return energy. This strategy achieves the same beam energy as a single long linac for one quarter the cost. Finally, specially arranged magnets allow the accelerating and decelerating beams to circulate in the same pipe, saving construction costs.

At its intended current of $40 \mathrm{~mA}$, CBETA would produce 100 -fold brighter beams than accelerators that don't use energy recovery. Georg Hoffstaetter, a researcher involved in the study who works at Cornell, says that a key potential application is using the electron beam to keep protons compressed at BNL's planned Electron-Ion Collider. Another application is generating an extreme ultraviolet source for computer-chip lithography.

This research is published in Physical Review Letters.

Jessica Thomas is the Editor of Physics. 